\title{
Author Index Vol. 31, Suppl 1, 1999
}

\section{European Surgical Research}

Aalbers, A.G.J. 39, 40, 42, 103

Aaltonen, V. 69

Aarsman, C.J. 3

Aase, S. 180

AbdEl-Aty, M. 144

Abdel-Ghaffar, T. 219

Abendroth, D. 162

Abou-Shady, M. 200

Abrahamse, S.L. 52, 66, 218

Abrego, D. 26, 27, 87

Adam, S. 77

Adamec, M. 137

Adawi, D. 80, 85, 101

Aeschbacher, B.C. 23

Agriantoni, M. 194

Ahonen, J. 70

Ahrné, S. 85, 101

Aigner, J. 231

Akasu, H. 120

Akedo, H. 199

Akira, S. 120

Aksoy, F. 213

Alam, H. 69

Albes, J. 81

Allen-Mersh, T.G. 68

Almeida, J.A. 87

Althaus, U. 82, 164, 136

Altomare, E. 75, 177

Aly, S. 144, 189

Ambacher, T. 21, 126

Ambalovs, G. 117

Amer, H. 189

Andersson, R. 184

Anding, W.J. 1

Androulakis, J. 194

Anelli-Monti, M. 10

Angeburger, M. 23

Antal-Szalmás, P. 160

Anwar, N. 151

Appelros, S. 207, 208

Appleton, S.G. 68

Araki, T. 120

Arima, S. 149, 188

Arkan, C. 126

Árkossy, P. 209, 221

Aruga, T. 168

Autikainen, T. 91

Autschbach, R. 11

Awane, M. 197
Ayalon, A. 222

Azzena, G. 128, 159

Babst, R. 231

Baccante, G. 76

Bach, O. 166

Baer, H.U. 13, 200

Bakay, A. 22

Baker, E.A. 40, 92, 158

Baldan, N. 152

Baldi, C. 133, 211

Balli, J. 26, 27

Balogh, À. 178

Balsiger, B.M. 1, 8

Banafsche, R. 157

Banic, A. 44, 111, 170, 180

Baqué, P. 62

Barbarisi, A. 210

Barker, J.H. 33

Baroncini, R. 63

Barr, C. 130

Barreiro, F. 192

Barrie, W.W. 118

Barros D'Sa, A.A.B. 9, 46

Batsis, Ch. 133, 134

Bauer, E.P. 11, 25

Baumhofer, J. 62

Baxter, G.F. 46

Becker, H. 190, 223

Becker, J.H.R. 165

Beckurts, K.T.E. 52

Beger, H.G. 94, 122

Behrens, R. 77

Beinhauer, B.G. 62

Bellamy, K. 119

Bellusci, R. 84

Bellón, J.M. 12, 71, 154

Belviranh, M. 213

Benchimol, D. 62

Berberat, P. 55, 63, 193

Berbig, R. 169

Berger, A. 108

Berger, J. 10

Bergin, F.G. 40, 72, 92, 158

Berke, G. 202

Bernard, J.L. 62

Bernardi, M. 75, 177

Berns, T. 77

Berrevoet, F. 155
Berry, D.P. 78, 118, 130, 206

Bertsch, Th. 233

Biberthaler, P. 39

Bicknell, G.R. 19

Biemer, E. 107

Biesterfeld, S. 97

Binz, K. 11

Biondi, R. 63

Birk, D. 122

Bischof, G. 96

Bischofberger, H. 79

Bittmann, I. 31

Bjelović, M. 225

Black, K. 13

Blanco, J.I. 88, 121

Blanco-Engert, R. 27

Bleese, N. 120

Blinman, T.A. 193

Boccardo, F. 109

Bockhorn, H. 27

Boerma, D. 77

Bohrer, T. 4

Borg, D.H. 109

Borgström, A. 207, 208

Börjesson, A. 184

Boros, M. 44, 175

Borrelli, V. 109

Borsiczky, B. 74, 170

Borst, C. 109

Bothur, P. 58

Bottino, D. 174

Bouchier-Hayes, D.J. 54

Bourgeon, A. 62

Bowley, D.M.G. 165

Brandes, H. 81

Brandt, C.T. 118, 184

Bráth, E. 22, 179, 223

Braun, F. 58

Breeman, W.A.P. 40

Brehm, W. 162

Breidenbach, W. 33

Breitenmoser, I. 92

Brenner, P. 32, 83, 152, 153

Brichon, P.Y. 226

Brighenti, L. 159

Broelsch, C.E. 96

Broucke, C.V. 84, 155

Browder, W. 85

Brown, D. 74

Bruch, H.-P. 163

\section{KARGER}

(C) 1999 S. Karger AG, Basel

Fax +41 613061234

E-Mail karger@karger.ch www.karger.com

Accessible online at

http://BioMedNet.com/karger 
Bruenagel, G. 42

Brune, I.B. 15

Brunnschweiler, D. 169

Brüwer, M. 3, 54, 102

Bráth, E. 22, 179, 223

$\mathrm{Bu}, \mathrm{X} .220$

Büchler, M.W. 8, 13, 55, 61, 63, $76,79,92,93,94,95,193$, 200, 207, 208

Bühler, M. 166

Buhr, H.-J. 101

Buhr, J. 151

Buján, J. 12, 71, 154

Bumbas̈irević, M. 228

Buriev, I. 221

Büscher, Ph. 229

Butinar, J. 216, 217

Butters, M. 71, 146

Butz, R.L. 101

Buxbaum, P. 234

Cainzos, M. 192

Calabrese, F. 152

Calderón, T. 29

Cama, A. 210

Cameselle, J. 192

Campisi, C. 109, 230

Caraceni, P. 75, 177

Carbajo, M.A. 88, 121

Carranza Ga ${ }^{\text {a }} 71$

Carrel, T. 26, 82, 164

Carrera, A. 71

Carvalho, G.L. 118, 184

Cass, M. 119

Castagliuolo, I. 96

Castagna, M. 63

Catena, F. 72, 75, 84, 158, 177

Cavallari, A. 72, 75, 84, 158, 177

Cavallari, G. 72, 75, 158, 177

Celic, B. 82

Celik, B. 136

Cetta, F. 133, 210, 211

Chaffanjon, P. 226

Chamuleau, R.A.F.M. 66, 218

Chan, J.K.Y. 82

Chau, G.Y. 203

Chen, B.-f. 7

Cheng, W. 188

Chi, C.W. 203

Chia, N.H. 143

Chick, W.K. 143

Chikamori, F. 135

Chillistone, D. 206

Chin, K.F. 54, 57, 189

Chooklin, S. 202

Christians, U. 58
Christov, V. 79

Chrzanowski, A.N.F.J. 24

Chung, T.K.M. 143

Cilley, J.H. 24

Clarke, D. 13, 105, 119

Clarke, D.L. 59

Clasper, J.C. 35, 165

Colizzi, C. 63

Conde, R. 192

Contreras, L.A. 12, 71

Conzelmann, A. 81

Cosentini, E. 96

Ćosić-Micev, M. 224

Csabina, S. 75

Csönge, L. 22

Curia, M.C. 210

Cuschieri, A. 15, 28, 88

Cvijanovic, R. 132

Czupryna, A. 129

Dai, X. 220

Dai, Y. 220

Dall'Agata, M. 75, 177

Dallman, M.J. 82

Danilov, M. 221

Dankelman, J. 16

Daoud, R. 105, 119

Dargai, O. 175

Datz, H. 27

Daum, S. 127

Davies, J.E. 78

Davis, P.A. 68

De Diego, J.A. 29

de Giorgi, L.B. 158

de Hemptinne, B. 53, 155

De la Torre, J. 29

De Laere, S. 155

De Nisi, A. 211

De Notariis, S. 72

De Vincentis, F. 84

de Wit, L.T. 16

Deakin, M. 187

Debatin, J.F. 105

Debus, E. S. 89

Delacuesta, M. 88, 121

Delius, M. 37

Delladetsima, I. 191, 192

DelRossi, A.J. 24

Demhartner, T.J. 59, 154

den Boer, K.T. 16

Dennison, A.R. 64, 65, 78, 118 , 206

Denzel, C. 86

Di Febbo, C. 76

di Mola, F.F. 93

Di Sebastiano, P. 76, 93

Diaz-E, J.A. 26, 27, 87
Diegeler, A. 11

Diem, S. 112

Dietl, K.-H. 155

Diller, R. 155

Dimakakos, P.B. 24, 136, 176

Djamaseb, A. 157

Djermanov, Z. 132

Dluzniewska, J. 100, 108, 204

Doenicke, A. 61

Dogaru, V. 218

Dohi, K. 73

Dolderer, J.H. 230

Dolfi, A. 63

Domenicali, M. 75, 177

Donath, S. 156

Donovan, D. 54

Doufas, A.G. 24, 136, 176

Dougems, D. 132

Doughman, T. 19

Drixler, T.A. 3

Drożd $\dot{z}$, W. 137, 138

Duda, E. 178

Duenes, J.A. 1

Dunjić, M. 228

Durowicz, S. 108

Duthie, G.S. 90, 100, 123, 124

Edelstein, J. 33

Eder, V. 83, 152

Egger, B. 8

Eggstein, S. 218

Ekelund, M. 201

El-Atrash, O. 189

El-Banna, M. 144, 189

El-Dessouky, M. 219

El-Ebeidi, G. 219

El-Ebidy, G. 99

El-Ebiedy, G. 151

El-Ella, K.A. 164

El-Enien, A.A. 151

El-Enin, A.A. 99

El-Fiky, A. 151

El-Hak, N.G. 99

El-Metwally, G. 17

El-Naggar, O. 219

El-Sefi, T. 219

El-Wahab, M. A. 151

Elhadad, A. 116, 148, 209

Elias, K. 58

Ellensen, V.S. 222

ElMeteini, M. 144

Emam, T.A. 15, 28, 88

Emmanuilidis, K. 87, 232

Emparan, C. 163, 185

Encke, A. 135

Enders, G. 173

Endrigkeit, S. 126 
Endzinas, Z. 147

Englert, A. 39

Entleutner, M. 87

Er, C. 213

Erhard, J. 52

Erli, H.J. 21

Erni, D. 44, 170

Ershov, V. 135

Ertel, W. 2

Evans, W. 105, 119

Exton, M.S. 156

Ezat, F. 151

Ezzat, F. 99

Faenza, A. 84, 158

Faist, E. 110

Falk, V. 11

Famulska, D. 129

Famulski, W. 129

Farhat, S. 104

Farkas, G. 214

Fathy, O. 99, 151

Faviana, P. 63

Favier, A. 226

Fawzy, A.T. 219

Fdez-Represa, J.A. 14, 29

Fedorov, V.D. 217

Feifel, G. 178

Fein, M. 93, 112

Felbinger, T. 83, 152

Felekouras, E.S. 191

Felix, R. 34

Fengler, Th.W. 143

Filimonov, V. 150

Filos, K. 132

Fink, U. 43

Fiorentini, G. 128

Fischer, H.P. 42

Fitzgerald, T. 69

Flautner, L.E. 204

Fleischmann, T. 232

Fleron, H. 70

Flügel, A.K. 1

Foitzik, T. 101

Fork, T. 90, 113

Fraitzl, C.R. 34, 59, 154

Frank, J. 223

Frank, T.G. 15, 28

Franken, J.P.M. 109

Franklin, M.E. 26, 27, 87

Freise, H. 35, 47, 114

Frey, C. 92

Frey, G. 226

Fricke, L. 163

Friedman, G. 117

Friess, H. 55, 61, 63, 76, 93, 95, 193, 200
Frilling, A. 96

Fuchs, K.-H. 89, 93, 112

Fujii, H. 199

Fujiu, T. 161

Fukai, M. 181

Fukuda, A. 94, 193

Fukuma, K. 73

Fukumoto, T. 67

Funakoshi, N. 183

Furian, L. 152

Furka, A. 160

Furka, I. 16, 22, 75, 160, 179, $205,214,221,223$

Furness, P. 19

Furukawa, H. 181

Futakawa, N. 234

Futami, K. 149, 188

Füzesi, L. 97

Gaber, A.O. 164

Gabriel, M. 139

Gadżijev, E.M. 216, 217

Gál, I. 27

Galavotti, D. 159

Galkowska, H. 86

Galuska, L. 205

Gamal, E.M. 16

Gansauge, F. 94

Gansauge, S. 94

Gantert, W.A. 18, 64, 114, 144

Ganz, R. 34, 154

García-Alonso, I. 162, 163, 185

García-Barreno, P. 186

García-Caballero, M. 88, 91, 112,121

García-Ceballos, A. 91, 121

García, J.C.A. 14

Gardiner, A. 90, 100, 123, 124

Gardovskis, J. 117, 142

Gasbarrini, A. 72

Gaskell, K. 54

Gawad, K.A. 232

Gebbink, M.F. 3

Gebhard, M.M. 176

Geishauser, M. 107

Genton, C.Y. 10

Georgescu, St. 150

Gerber, R. 94

Ghyczy, M. 44

Giannakenos, C. 227

Giger, R. 169

Gimeno, M.J. 12

Giordano, E. 158

Gippner-Steppert, C. 39

Girotto, A. 152

Glass, J.L. 26, 27, 87

Gloor, B. 55, 79, 193
Gogas, J. 191, 192

Gold, L.I. 93

Goldhahn, J. 166

Goldstein, S. 13

Golitsin, A. 154

Golling, M. 140

Góth, L. 160

Gouma, D.J. 16, 77, 98

Graber, H. 63, 93

Grajek, A. 139

Grass, R. 37

Grattagliano, I. 75, 177

Greenman, J. 54, 57, 189

Grigorov, G. 79

Grønbech, J.E. 180

Gross, T. 231

Gruber, S. 163

Gruca, Z. 205

Grünberger, T. 47

Gryglewski, A. 125

Gudat, F. 231

Guisasola, M.C. 186

Günerhan, Y. 111

Gürke, L. 60

Guzeeva, E. 221

Ha, T. 85

Habib, E. 116, 148, 209

Habler, O. 172, 173, 174

Habon, L. 185

Hacker, U. 61

Hagiwara, M. 196

Hahn, M. 38

Hahn, M.P. 169, 172

Haisa, M. 194, 195

Hak, N.G.E. 151

Halevy, A. 56

Hallek, M. 61

Hallén, M. 201

Halliday, M.I. 9, 46

Hamada, M. 194

Hammad, E. 17

Hammer, B. 229

Hammer, C. $32,83,152,153$, 173

Hankemeier, S. 21

Hanna, G.B. 28, 88

Hanselmann, R. 223

Hansen, S. 59

Hara, T. 5

Harder, F. 60

Harkin, D.W. 9, 46

Harmey, J. 54

Harris, A.G. 172

Hartley, J. 54, 57, 189

Hartman, R.J. 52

Hartmann, M. 48 
Hashi, H. 197

Hasse, C. 4, 102

Hathaway, D. 164

Hauck, M. 75

Haupt, W. 86

Hauser, S.P. 164

Hayashi, K. 161

Hayashi, T. 181

Heberer, M. 60, 231

Hehli, M. 166

Heidecke, C.-D. 87, 232

Heijnen, B.H.M. 98

Heise, W. 127

Heisterkamp, J. 149

Hejjel, L. 27

Hektor, J. 181

Helmy, A. 17, 219

Henneking, K. 151

Hensler, T. 15

Herderich, M. 112

Herfarth, Ch. 140, 157, 176

Herzog, B. 231

Hesse, U.J. 53, 84, 155

Hewitt, C.W. 24

Hierner, R. 108

Higashi, N. 196

Hiltebrand, L. 82, 111, 180

Hinoi, T. 73

Hinz, M. 32, 83, 152, 153

Hiraga, K. 125

Hirano, T. 81

Hirner, A. 42

Hirvonen, O. 69

Hofbauer, A. 110

Hofer, M. 15

Hoffmann, T. 175

Hofmann, A.F. 13

Hofstetter, W. 34, 154

Hohenberger, W. 86

Holland, T. 187

Hollingsbee, D. 222

Hölscher, A.H. 52

Holzinger, F. 13

Holzmann, B. 15, 232

Honda, O. 125

Hondroudaki, J.E. 176

Honduvilla G. ${ }^{\mathrm{a}}-$,N. 12,154

Hong, J. 185

Horiuchi, H. 181

Horsburgh, T. 130

Horvath, L.Z. 204

Horváth, L. 28

Hosokawa, T. 7

Hosseini, M. 58

Huber, H. 10, 32, 153

Hufländer, A. 232
Hungerbühler, R. 37

Hwang, J.S.T. 143

Ihling, C. 6

Iida, J. 181

Iino, Z.-I. 161

Ijzermans, J.N.M. 149

Ikai, I. 50

Ikeda, M. 73

Ikehara, S. 2

Imachi, K. 140

Imanishi, K. 140

Imaoka, S. 199

Imdahl, A. 6

Imeson, J. 89

Immer, F.F. 23, 26

Incze, D. 75, 160, 223

Inghammar, M. 184

Inkinen, K. 70

Innocenti, P. 76, 93

Inoue, T. 2, 198, 215

Intaglietta, M. 170

Inui, H. 2

Inui, K. 171

Ioannou, H. 133

Irén, M. 16

Ishikawa, H. 181

Ishikawa, O. 199

Ishikawa, S. 183

Ishikawa, Y. 65, 182

Ito, K. 37

Ito, S. 198

Ito, Y. 161

Itoh, S. 198

Iwasa, M. 115, 123

Iwasa, Y. 115, 123

Iwasaki, T. 67

Iwashita, A. 149, 188

Iwata, K. 196, 196

Iwata, S. 6

Iwazaki, S. 220

Izbicki, J.R. 232

Jaberansari, M.T. 46, 74, 185

Jabłońska, E. 129

Jacobi, C.A. 5, 66

Jacobs, B. 155

Jacobsen, N.O. 78

Jahnke, C. 140

Jain, S. 19

Jankowski, K. 205

Jankowski, T. 139

Janoušek, L. 137

Jedlinska, B. 48

Jeekel, J. 39, 42, 103

Jekić, I. 224, 225

Jenkins, T.D. 190
Jenkner, J. 6

Jensen, S.L. 78

Jeppsson, B. 80, 85, 90, 101, 113,201

Jevans, A. 33

Jin, M.B. 181

Jochum, M. 39

Jones, J.W. 33

Jönsson, K. 48

Jorgensen, J.J. 74

Jørgensen, U. 222

Juan, M.A.S. 14

Judich, A. 222

Jungraithmayr, W. 218

Jurado, F. 71, 154

Juretic, A. 231

Kabbara, S. 128

Kahan, B.D. 163, 185

Kairaluoma, M. 91

Kajiwara, T. 7

Kakisis, J. 191, 192

Kakita, A. 161

Kalbfleisch, J. 85

Kalechman, Y. 56

Kalinka, A. 81

Kalmuk, S. 49

Kalogeris, M. 131

Kalogezopoulou, C.P. 227

Kaminama, Y. 215

Kamiyama, Y. 2, 198, 215, 216

Kanai, F. 116, 119, 141

Kanazawa, A. 50

Kanje, M. 201

Kappas, A.M. 133

Kappelmayer, J. 160

Karapetian, I. 221

Karatzas, G. 191, 192

Karavias, D. 194

Karmazanovsky, G. 217, 221

Kasai, S. 56, 160, 201, 212

Kashiwagi, M. 55

Kaska, Ł. 205

Kasperk, R. 97, 126, 181

Kasprzak, P.M. 141

Kasravi, F.B. 80

Kaszaki, J. 175

Katic, V. 97

Kato, K. 56, 160, 201, 212

Katsube, T. 7

Katsura, N. 50

Kaun, M. 96

Kaur, G. 90, 100, 123, 124

Kawada, K. 6

Kawaguchi, Y. 215

Kawai, Y. 197

Kawashima, R. 194, 195 
Kawashima, T. 135

Kawashima, Y. 220

Kay, E. 54

Kazauresini, M. 227

Kehagias, J. 190

Keidar, S. 202

Keimer, R. 97

Keller, H. 169

Keller, N. 222

Kelm, C. 151

Kemming, G. 173, 174

Kendo, R. 205

Kenward, C.E. 74

Kerin, M.J. 57, 189

Késmárky, G. 185

Khonji, N. 105, 119

Kilian, M. 66

Kim, D. 69

Kimber, C. 88

Kimura, M. 194, 195

Kimura, W. 234

Király, Z. 25

Kirihara, Y. 5

Kirkpatrick, J. 69

Kirste, G. 218

Kishida, A. 181

Kiss, J. 16

Kitade, H. 215

Kitagawa, W. 120

Kitajima, M. 118, 184

Kitamura, Y. 120

Kitsakos, A. 133, 134, 135

Kiyoto, K. 196

Kjaergard, H. 70

Kjaergard, H.K. 222

Kjossev, K. 229

Klaebisch, G. 233

Klag, J. 145

Klar, E. 140, 157, 176

Kleeff, J. 61, 95

Kleen, M. 172, 173, 174

Kleine, P. 59

Klöppel, M. 107, 231

Klotz, A. 102

Klövekorn, W.P. 11, 25

Klüter, H. 163

Knez̈ević, J. 228

Knight, A. 19

Knoll, M. 77

Kobayashi, O. 127, 187

Kobori, O. 5

Koido, Y. 168

Köksal, N. 111, 169, 172, 225

Komborozos, V. 131

Komár, T. 179

Kon, M. 109

Kondo, T. 173
Köninger, J. 146

Konno, S. 7

Korc, M. 61, 93, 94, 193

Kore, M. 95

Kosaka, N. 5

Koseki, K. 168

Kosson, D. 32

Kostamoinen, L. 91

Kosti, P. 227

Kostopanagiotou, G. 176

Kosuna, K. 215

Kotsis, T.E. 24, 136, 176

Kouraklis, G. 191, 192

Kourias, E. 142

Kovács, I. 209, 221

Kovács, J. 75, 160, 179, 223

Kozaki, K. 20, 81

Kozaki, M. 20, 81

Kraas, E. 143

Krähenbühl, L. 13

Krapp, J. 61

Krasiński, Z. 139

Krasnik, M. 70

Kraus, Th. 140

Krauss, H. 139

Krejei, V. 44

Krejici, V. 82

Krenning, E.P. 40

Kreuch, R.G. 145

Krombach, F. 110

Kruft, S. 230

Ku, Y. 67, 68

Kubo, H. 197

Kujala, J. 70

Kukosh, M. 135

Kulig, J. 7

Kulli, Ch. 63

Kumada, K. 98, 99, 211, 212

Kumar, H. 57, 189

Kume, M. 65, 115, 182

Kunz, Ch. 229

Kupiee-Weglinski, J.W. 160

Kuriansky, J. 222

Kurochkina, A.I. 217

Kuroda, Y. 67, 68

Kurt, R. 111

Kusama, T. 199

Kusunoki, N. 67

Kuzume, M. 98, 99, 211, 212

Kwon, A.-H. 198

Laabs, S. 58

Laas, J. 59

Laato, M. 69

Laedrach, K. 228

Laffan, J. 85

Lagomarsini, B. 63
Laird, P.W. 93

Lameire, N. 53

Lang, R.D. 167

Lange, R. 52

Langer, M. 35

Längle, F. 47

Lantos, J. 27, 185

Lapatsanis, D. 131

LaRochelle, W.J. 56

Larsen, C.G. 78

Larsen, S.S. 222

Lasson, Å. 184

Laszuk, D. 108

Latouf, S. 46

Laurini, L. 133

Lausten, S.B. 78

Lázár, G. 178

Leaper, D.J. 40, 72, 92, 158

Leder, G. 94

Leder, S. 77

Lee, P.W.R. 124

Leunig, M. 34, 59, 154

$\mathrm{Li}, \mathrm{C} .85$

Li, J.-H. 202

Lill, C.A. 1

Lipkowski, A.W. 32

Lodi, A. 159

Löhe, F. 31

London, N.J. 78

Lorenz, M. 134, 135

Lorenz, W. 102

Loré, F. 133

Losanoff, H. 229

Losanoff, J. 229

Lu, Z. 63

Lueger, A. 10

Lui, W.Y. 203

Lukomska, B. 100, 108, 204

Lulchev, D. 79

Lyng, O. 180

Ma, K. 220

Makuuchi, M. 234

MacDonald, A. 54, 57, 189

Machida, H. 211

Mächler, H. 10

Mack, D. 232

Maddern, G.J. 64, 65

Maeda, C. 196

Maeda, K. 41

Maeda, T. 73

Maene, L. 155

Magata, S. 181

Mahler, F. 23

Mai, G. 55

Maier, M. 37

Maier, S. 87, 232 
Majcher, P. 125

Majstorovic, L. 97

Makarewicz, W. 205

Maksan, S.-M. 176

Maksymowicz, M. 30, 32

Maldonado, C. 33

Mallinder, P.A. 92

Mansel, R. 105, 119

Månsson, F. 113

Månsson, P. 90

Mao, Y. 80

Margaritis, V. 190

Mariani-Costantini, R. 210

Markon, I. 202

Markouizos, G. 133, 134

Marquet, R.L. 39, 40, 42, 103

Marra, S. 24

Marti, W.R. 169

Martignoni, M.E. 63, 93, 94

Martin, F. 88, 121

Martin, J.I. 88, 121

Martinez-Moreno, J.M. 91

Martinez-Moreno, M. 112

Martinov, M. 150

Martinovitz, U. 222

Martinsons, A. 142

Marty, B. 23

Marubayashi, S. 73

Maruta, M. 41

Maruyama, H. 95

Maruyama, K. 125

Marwan, I. 17, 219

Marx, A. 60

Marzola, M. 128

Matesz, K. 160

Maticic, D. 97

Matsui, Y. 215, 216

Matsumiya, A. 98, 99, 211, 212

Matsumoto, T. 98, 99, 211, 212

Matsumoto, Y. 199

Matsuno, N. 20

Matsuo, K. 125

Mattei, A. 60

Matthews, J.B. 96

Matuura, Y. 140

Matzen, K. 43

Matziolis, G. 181

Mayol, J. 14, 29

McCaigue, M.D. 46

McCarthy, J. 46

McCartney, J. 97

McNamara, D.A. 54

Mecklin, J.-P. 91

Meester, D. 84

Mehrabi, A. 140

Meier, J. 174

Meisner, F. 172, 173, 174
Meléndez, S. 112

Mellström, Å. 48

Mena, E. 192

Mendez, J. 162, 163, 185

Menger, M. 178

Menger, M.D. 49, 50, 51, 106, 182

Merlino, G. 56

Messmer, K. 31, 49, 110, 172, $173,174,175$

Metzger, U. 169

Meuli-Simmen, C. 105

Meyer, V.E. 105

Mica, L. 2

Micev, M. 224

Michov, R. 229

Mikó, I. 22, 75, 160, 179, 214, 223

Miller, S. 30

Milosevic, P. 132

Milz, S. 37

Minacci, C. 211

Minor, T. 18, 42, 49, 50, 51

Mirkovic, M. 132

Mironov, M. 229

Mirra, L. 194

Mito, M. 56, 201

Mitsui, T. 183

Miura, K. 227

Moberg, A.-C. 146

Mohr, F.W. 11

Mola, F. 76

Molin, G. 80, 85, 101

Moll, F. 11

Monson, J.R.T. 57, 124, 189

Montalto, G. 133, 210, 211

Montgomery, A. 146

Morgese, G. 210

Morishita, Y. 220

Morita, H. 2, 215

Morris, R.E. 136

Morsiani, E. 128, 159

Moríñigo, M.A. 112

Motohashi, H. 187

Motohasi, H. 127

Mourikis, D. 136

Mueller, A.R. 45

Mueller, C.A. 55

Mueller, X.M. 10

Müftüoğlu, T. 111

Mühlbacher, F. 47

Muhr, G. 38, 169, 172, 225

Mulcahy, K. 118

Muller, C.A. 46

Mun, E.C. 96

Muñoz, M. 91

Munzinger, U. 36
Muratsubaki, R. 161

Mussack, T. 39

Muto, T. 234

Mutschler, W. 223

Müller, A. 190

Müller, C. 31, 208

Müller, C.A. 207, 208

Müller, Ch. 79

Müller, F.P. 127

Müller, J.M. 5, 66

Müller, X. 23

Müller-Höcker, J. 83, 152

Naef, M. 8

Nagahama, T. 2

Nagao, T. 20, 81

Nagashima, T. 196

Nagel, E. 156

Nagy, P. 16

Nagy, S. 175

Nakagawa, A. 216

Nakamura, N. 2

Nakamura, Y. 197

Nakano, H. 211

Nakase, Y. 216

Namihira, T. 118, 184

Nande, G.A. 115

Naomoto, Y. 194, 195

Nardo, B. 72, 75, 84, 158, 177

Naritaka, Y. 7

Naumann, A. 231

Neef, H. 218

Nehéz, L. 28, 117

Nejdfors, P. 201

Nekarda, H. 43

Neuhaus, P. 45

Nguyen, W. 69

Nicholson, M.L. 19

Nicolaus, B. 82, 136, 164

Nienaber, Chr. 25

Nier, H. 126

Niinikoski, J. 69

Nikolić, J.A. 224

Nikolopoulou, V. 190

Nishitai, R. 50

Nobiling, R. 157

Nüssler, A.K. 94

Nüssler, N.C. 45

Nuzzo, G. 211

Nygaard, H. 59

Nyárády, J. 170

O'Brien, J. 45

O'Hara, R. 54, 57, 189

Oda, M. 2, 198, 215

Odze, R. 190

Ogata, K. 181 
Ogawa, K. 7

Ogawa, T. 220

Ogoshi, S. 115, 123

Ohigashi, H. 199

Ohmori, Y. 115

Ohshima, N. 183

Ohtani, N. 1

Ohwada, S. 220

Okabe, H. 50

Okada, K. 73

Okamoto, H. 199

Okamura, R. 196

Okumura, T. 198, 215

Oláh, A. 75, 160

Oláh, J. 178

Olszewski, W.L. 30, 32, 86, 100, 108, 167, 204

Onishi, K. 116, 119, 141

Onizuka, M. 183

Onodera, K. 56, 160, 201, 212

Opie, L.H. 46

Orda, R. 202

Ordemann, J. 5

Orth, K. 122

Ortiz-Oshiro, E. 14, 29

Orton, Ch. 13

Oshiro, Y. 73

Osman, M.O. 78

Oszkinis, G. 139

Ott, K. 43

Paar, O. 21

Pafiti, A. 24, 136

Pahlke, H. 143

Palmes, D. 21, 35, 47, 114

Panck, J. 137, 138

Papalabros, E. 191

Pape, A. 172, 173, 174

Pappas, J. 30

Paraschou, P. 134, 135

Parise, P. 152

Parker, S.J. 74

Pascual, G. 12, 154

Pasini, P. 72

Pasquinelli, G. 158

Patrinou, V. 132

Patti, M.G. 18, 64, 114

Pattyn, P. 53

Paulsen, P. 59

Pavars, M. 142

Pavez, C.O. 107

Pavlicek, M. 136

Pazzi, P. 159

Pedersen, J.H. 70

Peltonen, J. 69

Penka, I. 127

Pereira, J.G. 162
Perejaslov, A. 202

Pérez-Miranda, E. 91

Pernthaler, H. 76

Perotti, C. 20

Perthel, M. 59

Pes̈ko, P. 224, 225, 228

Peters, J.H. 93

Petersson, U. 208

Petö, K. 75, 205, 223

Petsas, T. 132, 227

Pfammatter, J.P. 26

Pfeffer, K. 87

Pierrefite, V. 62

Pingitore, R. 63

Piotrowski, Z. 129

Piperopoulos, P. 131

Pitschner, H.F. 25

Platz, K.-P. 45

Plenz, G. 21

Plesa, C. 150

Plusczyk, T. 178

Poch, B. 122

Podzuweit, T. 11

Pokrotnieks, J. 117

Polanski, J. 100, 204

Pollard, C. 78

Polymeneas, G. 142

Pommer, A. 38, 169

Popiela, T. 7, 125, 129

Portugal, V. 162

Post, S. 233

Potel, J. 192

Pothoulakis, C. 96

Prati, U. 20

Prizov, V. 221

Probst, A. 21, 35

Provido, H. 69

Ptak, W. 125

Puchkov, K. 150

Pukacki, F. 139

Pulawska, T. 222

Puviani, A.C. 159

Qiu, F. 220

Quervain, I.K. 36

Raab, S. 172

Raffaelli, N. 133, 210, 211

Raftopoulos, J. 191

Rahn, B. 229

Rampp, T. 102

Rango, C. 210

Rashed, M.Y.T. 219

Rauen, U. 52

Raveh, J. 228

Ray, M. 33

Reber, H.A. 55, 193
Redl, H. 234

Rees, J. 105, 119

Rees, Y. 206

Refior, H.J. 37, 110

Regaert, B. 84

Rehak, P. 10

Reichart, B. 32, 83, 152, 153

Reichenspurner, H. 32, 61, 83, 152,153

Reill, P. 230

Reimuth, S. 145

Reinshagen, K. 6

Reuter, S. 30

Reuthebuch, O. 11

Reyerkerk, A. 3

Reyes, R.M. 192

Reynders, P. 165

Ribenieks, R. 142

Richard, M.J. 226

Richter, A. 233

Richter, S. 18, 49, 50, 51

Riegler, M. 96

Riese, J. 86

Riesener, K.P. 97, 181

Riess, A.G. 120

Riess, F.-Ch. 120

Rigler, B. 10

Rigotti, P. 152

Riis, C. 59

Ringe, B. 58

Rinkes, I.H.B. 3

Ritchie, E.D. 3

Rius, F. 91, 112

Robertson, G.S.M. 64, 130, 206

Roda, A. 72

Rogy, M.A. 62

Röher, H.D. 127

Rohr, M. 126

Rokitansky, A.M. 234

Romanowski, B.J. 105

Rosen, D. 202

Rösken, F. 106

Roth, E. 47

Röth, E. 27, 170, 185

Rõth, E. 46, 74

Roth, M. 11

Rothmund, M. 4, 102

Roukos, D.H. 133, 134, 135

Roveda, L. 20

Royle, P. 92

Rozsos, S.E 147

Rubin, J.S. 56

Rucker, A. 83, 152

Rücker, M. 106

Ruf, G. 6

Ruiz, A. G. 162

Ruiz, J. 112 
Rumpf, C. 167

Rusev, D. 79

Russ, M. 71

Rustgi, A.K. 190

Rüttinger, D. 49

Rybinsky, A. 135

Rydning, A. 180

Ryska, M. 137

Sabbas, A. 47

Sabljak, P. 225, 228

Sadek, A. 219

Şahin, M. 213

Sailer, M.A. 89

Sairenji, M. 127, 187

Saito, M. 127

Saitoh, M. 187

Sakai, H. 98, 99, 170, 211

Sakakura, Y. 2

Sakamoto, I. 161

Sakamoto, T. 168

Sakata, H. 56, 160, 201, 212

Sakitani, K. 198

Sakurai, E. 20

Salakou, S. 191

Salvaneschi, L. 20

Sánchez, M.J. 186

Saner, H. 23

Santoni, B. 72, 75, 84, 158, 177

Sápy, P. 209, 221

S̈aranović, D. 228

Sarr, M.G. 1, 8

Sato, K. 161

Satoh, S. 50

Satoh, Y. 220

Satoi, S. 2, 215

Savio, M. L. 152

Savvina, T. 221

Sawano, T. 135

Schäfer, R. 77

Schäffer, F. 140

Schaffner, Th. 136

Schauer, R. 49

Schedlowski, M. 156

Schelzig, H. 162

Scheule, A.M. 30

Scheyer, M. 146

Schildberg, F.W. 31, 49, 110

Schildhauer, T. 172, 225

Schimke, I. 66

Schindl, M. 47

Schleimer, K. 52

Schlüter, M. 233

Schmid, K.W. 3, 54, 102

Schmid, S. 8

Schmidt, E. 75, 223

Schmidt, W.U. 127
Schmitter, N. 193

Schmitz-Rixen, Th. 141

Schmoeckel, M. 32, 83, 152, 153

Schneider, C. 232

Schneider, E. 1

Schnelldorfer, T. 94

Schnider, A. 169

Schröder, D. 145

Schröder, M. 94

Schroeder, T. 105

Schteingart, C.D. 13

Schult, M. 156

Schulze, C. 61

Schumann, B. 204

Schumpelick, V. 97, 126, 181

Schürmann, G. 3, 54, 102

Schuster, K.L. 233

Schütz, E. 58

Schwaiger, M. 43

Schwemmle, K. 151

Sckell, A. 34, 59, 154

Scola, E. 166

Scopa, C.D. 190

Scott, E.M. 72

Šefr, R. 127

Seidel, D. 83, 152

Seiler, A.M. 23, 26

Senninger, N. 3, 54, 102, 155

Serfözö, J. 160

Shabtai, M. 222

Shah, K. 131

Shahsafaei, A. 190

Shawki, A. 219

Shen, Z.-L. 108

Shi, M.-S. 97

Shibata, D. 93

Shibuya, S. 135

Shimahara, Y. 65, 182

Shimamura, T. 181

Shimazaki, Y. 171

Shimazu, M. 118, 184

Shimizu, K. 120, 197

Shimura, H. 211

Shipkova, M. 58

Shipuliova, I.V. 217

Shirakawa, Y. 194, 195

Shokouh, A. 164

Shrikhande, S.V. 115

Siedlar, M. 129

Siewert, J.R. 15, 43

Sigala, F. 191

Sigurdsson, G.H. 44, 82, 111, 180

Simić, A. 224, 225, 228

Simon, F. 162

Simoncini, M. 84

Simpson, A.H.R.W. 35
Simpura, E.-M. 91

Simunek, M. 234

Sipka, S. 160

Sitter, H. 102

Skinner, K.A. 93

Skrekas, G. 131

Skwara, W. 25

Sledziński, Z. 205

Slooter, G.D. 40

Smeds, C.-G. 70

Sogukoglu, T. 96

Sojar, T. 216

Solecki, R. 129

Solimann, T. 47

Sosef, M.N. 66, 218

Sotoda, Y. 171

Spiegel, H.U. 21, 35, 47, 114, 155

Spini, D. 133

Spisni, R. 63

Sredni, B. 56

Staikov, P. 79

Stamatiades, A. 142

Stange, B. 45

Stangl, M. 61

Stanisavljevië, D. 216, 217

Stark, G. 10

Stassen, H.G. 16

Staudenmaier, R. 231

Steinau, H.U. 225

Steininger, R. 47

Steinkuhl, R. 77

Stepkowski, S. 163

Stocker, F. 26

Stockham, G. 15, 28

Stojakov, D. 225, 228

Storck, M. 162

Straatsburg, I.H. 77, 98

Stratan, I. 150

Strik, M.W. 163

Strobel, O. 106

Strotmann, C. 30

Stupnicki, A.F. 92

Sturlan, S. 62

Stüssi, E. 36

Stylianides, G. 133, 134

Suárez, A. 186

Sueda, T. 140

Sugimoto, K. 168

Sugimoto, T. 171

Sultan, A. 151

Sun, Z.W. 184

Sutter, P.-M. 60

Sutton, C. 131

Sutton, C.D. 78, 89, 118, 130, 206

Suzuki, T. 181 
Sweetland, H. 105, 119

Szabó, Gy. 160, 223

Szakács, J. 178

Szalai, H. 27

Szalay, L. 175

Szalay, Z.A. 25

Szántó, Z. 170

Szanyi, A. 205

Szarzynski, M. 218

Szczepanik, A.M. 129

Szczepanik, M. 125

Szczesny, G. 167

Szentmiklósi, A.J. 75

Szira, P.T. 147

Tachikawa, D. 149, 188

Tadokoro, F. 161

Takabayashi, A. 6, 197

Takahama, T. 116, 119, 141

Takahashi, T. 161, 171

Takahashi, Y. 161

Takakuwa, T. 196

Takamatsu, M. 67

Takase, Y. 135

Takatsu, K. 120

Takayama, H. 56

Takayanagi, K. 168

Takazawa, Y. 161

Takemura, K. 196

Takeuchi, H. 81

Takeyama, O. 50

Takeyoshi, I. 220

Taki, Y. 197

Tam, P.K.H. 82, 188

Tanahashi, Y. 220

Tanaka, I. 196

Tanaka, N. 194, 195

Tanaka, S. 120

Tang, W. 193

Taniguchi, M. 181

Tatem, L.D. 24

Tcholakov, O. 55, 79

Teitz, S. 56

Teleky, B. 96

Tepetes, K. 194

Terajima, H. 173

Tevaearai, H.T. 10

Thein, E. 172

Theodorou, D. 61

Theodosopoulos, Th. 142

Thiäner, A. 173

Thiede, A. 89

Thoclaclus, H. 113

Thomas, S. 11

Thompson, J.N. 68

Thomson, S.R. 59
Thorlacius, H. 90, 182

Tihanyi, T.F. 28, 117, 204

Tillmanns, J. 174

Tischer, T. 37

Todo, S. 181

Todorović, V. 224

Toledano, M. 88, 121

Tombach, B. 77

Tominaga, M. 67

Tomoyasu, S. 183

Ton-Nu, H.-T. 13

Topping, K. 54, 57, 189

Tošenovský, P. 137

Tóth, P. 221

Toti, P. 133

Toval, J.A. 91, 112

Trentz, O. 2

Triantafyllou, A. 191

Troisi, R. 53, 84, 155

Trotovsek, B. 216

Trotta, F. 20

Tsai, A.G. 170

Tsamandas, A.C. 190, 191, 194

Tse, C.W.C. 143

Tsiligiris, B. 136

Tsuburaya, A. 127, 187

Tsuchida, S. 67, 68

Tsutsui, N. 140

Tu, W. 215

Tuma, J. 127

Turi, P. 72, 75, 84, 158, 177

Ubhi, S. 89

Uchikoshi, T. 196

Uchinami, H. 65, 182

Uchiyama, M. 20

Uesugi, T. 50

Uhl, W. 55, 79, 193, 207, 208

Ustuner, T. 33

Utsumi, T. 41

Uzun, M.A. 111

Vagianos, C. 132, 190, 227

Vajda, G. 185

Valente, M.L. 152

Valieri, L. 128, 159

Valsecchi, P. 20

van den Tol, M.P. 39

van Eijck, C.H.J. 39, 40, 42, 103

van Gulik, T.M. 52, 66, 77, 98, 218

van Hillegersberg, R. 149

van Noorden, C.J.F. 77

van Rossen, M.E.E. 39, 42, 103

van Runnard Heimel, P.J. 66

Vatansev, C. 213
Vatansev, H. 213

Veihelmann, A. 110

Veitch, P. 131,

Veitch, P.S. 89, 130

Velada, J. 222

Velitchkov, N. 229

Veljkovic, R. 132

Vendemiale, G.L. 75, 177

ven Rooijen, N. 232

Venturini, R. 152

Veres, L. 209

Vering, T. 77

Verreet, P.R. 127

Vesterweber, D. 182

Viarengo, G.L. 20

Viha, K. 131

Villagrasa, E. 91, 112

Villanueva, F.J. 186

Vinci, D. 128

Vintila, D. 150

Voest, E.E. 3

Vogel, U. 30

Vögeli, P. 164

Vogt, P. 225

Vollmar, B. 18, 49, 50, 51, 106, 182

von Dobschuetz, E. 175

von Donnersmarck, G.H. 110

von Flüe, M. 18, 64, 114, 144

von Meyenfeldt, E.M. 218

von Schulthess, G.K. 105

Von Segesser, L.K. 10, 23

Vukicevic, S. 97

Wahba, A. 17

Wahl, D. 37, 217

Wahl, M. 216, 217

Wakabayashi, G. 118, 184

Waldmann, E. 47

Walgenbach, K.J. 42

Walpoth, B. 58

Walpoth, B.H. 82, 136, 164

Walpoth, N. 82

Walsh, S. 13

Walsh, T.N. 54

Walter, G.F. 108

Walther, T. 11

Wang, J.H. 54

Wang, L. 94, 220

Wang, M. 85

Wang, X.D. 184

Wanner, G.A. 2

Wastell, C. 68

Watkins, P.E. 35, 74, 165

Watson, A. 104

Way, L.W. 18, 64, 114, 144 
Weber, W. 43

Wei, T. 2, 77

Weiner, R. 27

Weis-Fogh, U. 70

Weiss, G. 140, 176

Wemyss-Holden, S.A. 64, 65

Wendl, K. 233

Wenger, F.A. 66

Wenzl, E. 96

Werker, P.M.N. 109

Westermann, J. 156

Westermann, S. 178

Weström, B. 201

White, S. 131

White, S.A. 19, 78, 89, 118, 130, 206

Wiedemann, E. 39

Wiegand, N. 170

Wildhirt, S. 61

Wildi, S. 8, 61

Wildisen, A. 18, 64, 114, 144

Wilke, W. 15

Williams, D. 85

Williams, S.T. 19

Wimmer, C. 83,152

Winde, G. 3, 54, 155

Winslet, M.C. 104

Winternitz, T. 28

Wlk, M. 96

Wojtezyk, C. 174

Wolf, W. 25

Wolfárd, A. 44

Wong, W. 187

Wordliczek, J. 129
Woźniak, M. 205

Wu, C.W. 203

Wysocki, A. 137, 138

Xia, Z. 220

Yamagami, K. 65

Yamaguchi, M. 98, 99, 211, 212

Yamaguchi, S. 196

Yamamoto, A. 115

Yamamoto, H. 65

Yamamoto, T. 183

Yamamoto, Y. 65, 182

Yamamura, T. 196

Yamaoka, Y. 50, 65, 182, 197

Yamashina, S. 161

Yamashita, K. 181

Yamatsuji, T. 194

Yamauchi, J. 18, 49, 50, 51

Yanagi, K. 183

Yanagi, Y. 196

Yang, F. 220

Yannopoulos, P. 131

Yassin, M.M.I 9, 46

Yellon, D.M. 46

Yilmaz, O. 213

Yin, H. 7

Yin, S. 97

Yla-Outinen, H. 69

Yokota, R. 181

Yonezawa, K. 65, 182

Yoshida, K. 2, 212

Yoshikawa, T. 127, 187

Yoshito, H. 140
Young, C.V. 9

Young, I.S. 46

Yu, S.-C. 199

Yuan, R.H. 199

Zajac, L. 204

Zaleska, M. 30, 86

Zalewski, B. 129

Zantl, N. 87, 232

Zapalski, S. 139

Zapletal, C. 176

Zdichavsky, M. 33

Zedler, S. 110

Zembala, M. 129

Zhang, Z. 215

Zhao, B. 234

Zid, M.A. 99

Zied, M. A. 151

Zielke, A. 4, 102

Ziemer, G. 81

Zilli, A. 109

Zimmer, K.P. 102

Zimmer, M.M. 223

Zimmermann, A. 55, 63, 93, 94, 193, 200

Zimmermann, F. 43

Zimmermann, G. 146

Zimmermann, U. 4

Ziolkowska, A. 86

Zlatarski, G. 79

Zoricic, D. 132

Zu, Z. 94

Zuckermann, M. 210, 211 